\title{
VORURTEILSFORSCHUNG UND SOZIALSTRUKTUR
}

\author{
ZU EINEM FORSCHUNGSPROJEKT DES BERLINER ZENTRUMS \\ FÜR ANTISEMITISMUSFORSCHUNG
}

Obwohl die Studie von Alphons Silbermann ${ }^{1}$ im Hinblick auf ihre Methode kritisiert wurde, läßt sich als eines ihrer Ergebnisse festhalten, daß die Abwesenheit einer rechtlichen und sozialen Diskriminierung der jüdischen Bevölkerung in der Bundesrepublik Deutschland durchaus noch kein Verschwinden negativer Vorurteile gegen Juden und Judentum bedeutet. Silbermanns Erhebungen deuten auf einen beträchtlichen ,, latenten Antisemitismus" hin, nämlich das Fortleben einschlägiger Stereotypen, Fehlinformationen, biologischer Klischees. Ähnliches hat eine Analyse von Meinungsfragen für Österreich ergeben. ${ }^{2}$

Die geisteswissenschaftliche Forschung hat Stereotypen von Juden und Judentum in der Literatur, der Karikatur, der Malerei ermittelt. Die internationale Diskussion um die Texte von Passionsspielen hat die Aufmerksamkeit auf die Kontinuität von entsprechenden volksreligiösen Vorstellungen gelenkt. Auch in anderen sub-literarischen Texten wurden z.T. bis ins zwanzigste Jahrhundert fortlebende negative Stereotypen festgestellt. ${ }^{3}$ Die Fruchtbarkeit der Fragestellung, die sich auf die populären Judenbilder konzentriert, zeigt ferner die unter Verwendung kulturanthropologischer und historischer Methoden erarbeitete Studie von Utz Jeggle, in der die Überlieferung und Adaption entsprechender Vorstellungen in einem begrenzten Raum analysiert wird. ${ }^{4}$

Das Zentrum für Antisemitismusforschung der Technischen Universität

\footnotetext{
1 A. Silbermann, Sind wir noch Antisemiten?, Köln 1982.

$2 \mathrm{Vgl}$. B. Marin, ,,Ein historisch neuartiger, Antisemitismus ohne Antisemiten'? Beobachtungen und Thesen am Beispiel Österreichs nach 1945", in: Geschichte und Gesellschaft, Jg. 5 (1979), S. 545-67.

3 Vgl. H. A. Strauss, ,Pre-Emancipation Prussian Policies towards the Jews”, in: Leo Baeck Institute, Year Book, Bd 11 (1966), S. 107-36 (für die preußischen Regierungsakten des Vormärz); ders., , ,Die preußische Bürokratie und die anti-jüdischen Unruhen im Jahre 1834", in: Gegenwart im Rückblick, hrsg. von H. A. Strauß und K. R. Großmann, Heidelberg 1970, S. 27-55.

4 U. Jeggle, Judendörfer in Württemberg, Tübingen 1969.
} 
Berlin sieht in diesen noch unzulänglich erfaßten und beschriebenen Erscheinungen ein Problem der Grundlagenforschung zum Vorurteil in Geschichte und Gegenwart. Es beabsichtigt, durch eine von verschiedenen wissenschaftlichen Ansätzen ausgehende (kon-disziplinäre) Forschungsarbeit ,,Geschichte und Wandlung der Bilder von Juden und Judentum in der sub-literarischen und ikonographischen Tradition (popular culture) im Zeitraum von 1815 bis 1850 " zu erfassen, um so eine zusätzliche Dimension des modernen Antisemitismus zu erschließen und weitere Arbeiten in dieser Richtung anzuregen. Die zentrale Arbeitshypothese, die empirisch verifiziert werden soll, läßt sich folgendermaßen formulieren.

Dem Antisemitismus, der - in Theologie, Literatur, politischer Ideologie, den Wissenschaften - einen Teil der bildungsbürgerlichen Kultur ausmachte, korrespondierten gewisse - konstante oder sich wandelnde Bilder von Juden und Judentum in der popular culture. Diese volkstümlichen Vorstellungen begünstigten - so lautet die Vermutung - die massenhafte Rezeption bzw. Duldung des modernen Antisemitismus.

Es handelt sich hierbei um eine Problematik von gesamteuropäischem Ausmaß, die indessen in den verschiedenen Ländern und Regionen von der jeweiligen Entwicklung der ökonomischen, sozialen, rechtlichen Verhältnisse, nationalen Eigenarten, den spezifischen religiösen, kulturellen, politischen Traditionen bestimmt wurde. Aus forschungspraktischen Gründen konzentrieren wir uns vorerst auf den deutschen Sprachraum. Ausgewertet werden - außer den Quellen der allgemeinen und Religionsgeschichte - die gedruckten und ungedruckten Quellen der Regionalgeschichte und Volkskunde, die Traktat- und Trivial-literatur sowie daß Bildmaterial der populären Graphik und Karikatur.

Als Grundsatz gilt, daß die Antisemitismusforschung nicht von den geschichtlichen Zusammenhängen abstrahieren kann. Der jeweilige Inhalt des Vorurteils und seine Träger, wirtschaftliche und soziale Krisen wie displacements sind für die Erklärung der konkreten Erscheinungsformen des Antisemitismus ebenso relevant wie politische Ereignisse und Ideen. Die bisherigen Arbeiten zur Geschichte des Antisemitismus haben diese Aspekte, je nach Quellenlage und Interessenrichtung, unterschiedlich stark belichtet. Aber der Schwerpunkt dieser Literatur liegt auf der Zeit nach 1871 und hier auf der Geschichte des organisierten Antisemitismus.

Es wird ferner davon ausgegangen, daß es die konkreten Erscheinungsformen des Antisemitismus jeweils auf die Ereignis- und Strukturgeschichte der jüdischen Gruppe zu beziehen gilt. Der moderne Antisemitismus ist eine Begleiterscheinung der Integration (,Akkulturation") der jüdischen Gruppe; außer negativen gab es auch positive Bilder und Traditionen, außer Antagonismus gab es auch Kooperation. 
Richwarmerischer Blick in die Some

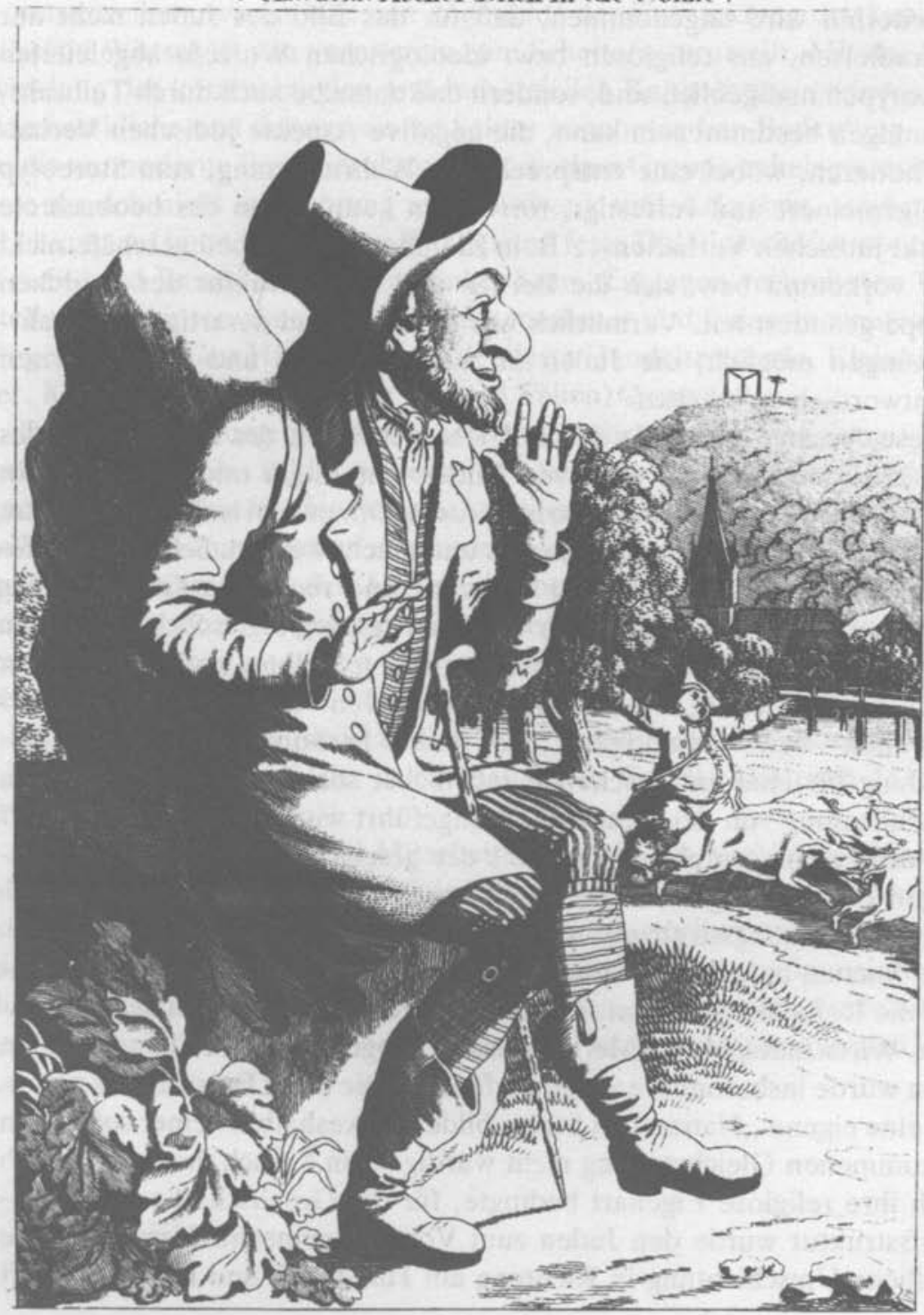

Gotts Wunder weleher Glanz und Schein;

Das mul's ep's rores von Verôilding sevn!

Eines von zahllosen Beispielen antijüdischer Stereotypen in der populären Graphik: ,,Schwärmerischer Blick in die Sonne”, Kupferstich (um 1820), Germanisches Nationalmuseum, Nürnberg 
Weiterhin wird angenommen, daß für das Bild des Juden nicht nur die tradierten, aus religiösen bzw. ideologischen Wurzeln abgeleiteten Stereotypen maßgeblich sind, sondern daß dasselbe auch durch Teilwahrnehmungen bestimmt sein kann, die negative Aspekte jüdischen Verhaltens notieren, wobei eine entsprechende Wahrnehmung, zum Stereotyp verallgemeinert und verfestigt, fortwirken kann, wenn der beobachtete Aspekt jüdischen Verhaltens, z.B. in Kleinhandel oder Leihgeschäft, nicht mehr vorkommt bzw. sich die Berufs- und Sozialstruktur der jüdischen Gruppe geändert hat. Vermutlich war es auf Grund derartiger Teilwahrnehmungen möglich, die Juden für wirtschaftliche und soziale Krisen verantwortlich zu machen. ${ }^{5}$

Diese Ansätze signalisieren die Vielschichtigkeit der Problematik des Antisemitismus wie der positiven Bilder von Juden und Judentum. In unserem Projekt soll daraus eine historische Dimension isoliert und für die Zeit der Früh-Emanzipation genauer untersucht werden. Selbst die philosophes der Aufklärung, die für Toleranz und rechtliche Gleichstellung der Juden plädierten, ließen sich von einer fragwürdigen Sicht von deren Religion, wirtschaftlichen Betätigung, sozialen Stellung und körperlichen Beschaffenheit leiten. Was von ,Konservativen" als Zeichen der ,Verworfenheit" bzw. ,Niedrigkeit" der Juden perhorresziert und zur Begründung für eine entsprechende rechtliche, soziale und wirtschaftliche Ausschliessung von der Gesellschaft angeführt wurde, war für ,liberale" Beobachter ein komplexes Ergebnis der geschichtlichen, verbesserungsfähigen Entwicklung.

Die ,Erziehungsstrategie” der Emanzipationsgesetzgebung der deutschen Staaten beruhte auf einem negativen Urteil über Juden und Judentum, die Rolle der Juden im Agrarstaat, ihre Bedeutung für die absolutistische Wirtschaftspolitik (Merkantilismus, beginnender Freihandel). Den Juden wurde insbesondere vorgeworfen, daß sie einer Binnenethik anhingen, eine eigene ,Nation" im Staate bildeten, weshalb sie einer sofortigen vollkommenen Gleichstellung nicht würdig seien. ${ }^{6}$ Auch ihre - angeblich durch ihre religiöse Eigenart bedingte, für die Gesellschaft schädliche Berufsstruktur wurde den Juden zum Vorwurf gemacht. Versuche, ihre berufliche Umschichtung in Richtung auf Handwerk und Landwirtschaft

\footnotetext{
${ }^{5} \mathrm{Vgl}$. hierzu etwa für die Wirtschaftskrise von 1873 bis $1895 \mathrm{H}$. Rosenberg, Große Depression und Bismarckzeit. Wirtschaftsablauf, Gesellschaft und Politik in Mitteleuropa, Berlin 1967.

6 Zur etappenweisen Gleichstellung und der damit verbundenen Beibehaltung des Sonderstatus für Juden mit seinen negativen sozialen und politischen Folgen vgl. für die Emanzipationsperiode ausführlich R. Rürup, Emanzipation und Antisemitismus. Studien zur ,,Judenfrage”' der bürgerlichen Gesellschaft, Göttingen 1975.
} 
voranzutreiben, waren indessen angesichts der Entwicklung der kapitalistischen Wirtschaft von vornherein zum Scheitern verurteilt. Mit der Entwicklung des kommerziellen und industriellen Kapitalismus verloren der handwerkliche und der agrarische Sektor zunehmend an Bedeutung. Wie es die vormoderne Berufsstruktur der jüdischen Gruppe nahelegte, schichtete sie sich nicht in Richtung auf Handwerk und Ackerbau, sondern in Richtung auf konzessionierten Handel und feste Detailgeschäfte sowie das Kredit- und Bankwesen (Privatbanken) um. Entgegen verbreiteten Vorstellungen wurden aber nicht Großunternehmer und Finanzmagnaten die Regel, sondern aus Hausierern wurden feste Handelsreisende, Kleinhändler, Kleinagenten oder (in den meisten Fällen) Angestellte.

Diese Entwicklung ging zum Teil der rechtlichen Gleichstellung voran und beeinflußte das Bild des Juden, das den Überlegungen der Regierungen und den Debatten der Parlamente über Wünschbarkeit und ,,Zeitgemäßheit" der Emanzipation zugrundelag. Gleichzeitig wurde die traditionelle Wahrnehmung ,der Juden" als homogener Gruppe infolge der sozialen Differenzierung immer mehr zum Stereotyp bzw. durch die negativen Assoziationen zu einem auf unzureichender Gewichtung der Tatsachen beruhenden Vorurteil. Das Bild des Juden, wie es sich bereits vor der ,,Reaktionszeit" herauskristallisiert hat, enthält die charakteristischen ,,Ungleichzeitigkeiten”, die in der deutschen Gesellschaft nach $1815 \mathrm{zu}$ erkennen sind.

Das Projekt wird von vier Mitarbeitern bearbeitet, die die spezifischen Methoden und Ergebnisse ihrer Disziplinen verwenden: einer Literaturhistorikerin, einem Sozialhistoriker, einem Kunsthistoriker und einem Religionshistoriker. Die Stiftung Volkswagenwerk, Hannover, finanziert drei dieser Stellen über eine Laufzeit von drei Jahren, die Deutsche Forschungsgemeinschaft, Bonn, eine Stelle über zwei Jahre. Die wissenschaftliche Leitung liegt bei mir. 